\title{
Construção e validação fatorial da Escala de Caracterização do Burnout (ECB)
}

\author{
Mauricio Robayo Tamayo \\ Bartholomeu Tôrres Tróccoli \\ Universidade de Brasília
}

\begin{abstract}
Resumo
Este artigo descreve o desenvolvimento da Escala de Caracterização do Burnout (ECB). No primeiro estudo, 375 trabalhadores de enfermagem e do ensino responderam a primeira versão da ECB com 77 itens. Análises fatoriais revelaram os fatores Exaustão Emocional (Alfa =0,94); Desumanização (Alfa $=0,88)$, Realização Pessoal (Alfa = 0,76) e Decepção no Trabalho (Alfa =0,80). No segundo estudo, 787 membros da Policia Civil responderam a segunda versão da ECB com 46 itens. Os resultados de novas análises fatoriais evidenciaram os mesmos três fatores do estudo 1: Exaustão Emocional (Alfa $=0,93$ ); Desumanização (Alfa $=0,84)$ e Decepção no Trabalho $($ Alfa $=0,90)$. Os itens do fator Realização Pessoal, observado no estudo 1, foram incorporados ao fator Decepção no Trabalho. A versão final da ECB apresentou 35 itens. O fator Exaustão Emocional evidenciou a maior consistência interna. Os níveis de consistência interna dos outros dois fatores superaram índices apresentados por outras medidas de burnout.
\end{abstract}

Palavras-chave: burnout; avaliação; estresse ocupacional.

\begin{abstract}
Construction and factorial validation of the Burnout Characterization Scale (ECB). This paper presents the development of the Burnout Characterization Scale (ECB). In the first study, 375 nurses and teachers answered the 77 items initial version of the ECB. Factor analysis revealed the factors Emotional Exhaustion $($ Alpha $=0.94)$; Dehumanization $($ Alpha $=0.88)$, Personal Achievement $($ Alpha $=0.76)$ and Disappointment in the Work $($ Alpha $=0.80)$. In the second study, 787 members of the police force answered the second 46 items version of the ECB. Factor analysis results showed the same three factors revealed in the first study: Emotional Exhaustion (Alpha $=0.93$ ); Dehumanization $($ Alpha $=0.84)$ and Disappointment in the Work $($ Alpha $=0.90)$. The items of the factor Personal Achievement found in the first study were included in the factor Disappointment in the Work. The ECB's final version has 35 items. The factor Emotional Exhaustion had the greatest internal consistency. The internal consistencies of the two other factors were greater than the ones found in other measures of burnout.
\end{abstract}

Keywords: burnout; assessment; occupational stress.

$\mathrm{O}$ burnout é definido como uma síndrome psicológica decorrente da forte tensão emocional crônica associada ao estresse ocupacional severo (Byrne, 1993a, 1993b; Mallet, Jurs, Price, \& Slenker, 1991; Meier, 1983, 1984; Silverstein, 1982). As primeiras manifestações da síndrome do burnout foram observadas em membros de profissões cujo caráter assistencial (enfermeiros, professores, policiais, terapeutas) implica em relacionamentos freqüentes e diretos com pessoas que precisam de algum tipo de cuidado e/ou ajuda (Maslach, 1978, 1993; Maslach \& Jackson, 1986; Maslach \& Leiter, 1997; Maslach, Schaufeli, \& Leiter, 2001). As manifestações da síndrome do burnout incluem perda progressiva do idealismo, das expectativas, da energia, da satisfação e do comprometimento no trabalho e indica a presença de um autoconceito negativo e atitudes desfavoráveis em relação ao trabalho e às pessoas que se encontram dentro do ambiente laboral, tais como clientes, colegas e chefia (Brill, 1984; Craig, 1986; Edelwich \& Brosky, 1980; Etzion \& Pines, 1986; Garden, 1989, 1991; Leiter \& Meechan, 1986; Meier, 1983).

Segundo Tamayo (2002), desde o início da década de 1970 as investigações sobre a síndrome do burnout têm revelado entre seus correlatos, concomitantes e possíveis conseqüências, aspectos como: distúrbios individuais (depressão, queixas psicossomáticas, problemas de saúde, uso de drogas), atitudes inadequadas (insatisfação no trabalho, falta de comprometimento organizacional, intenção de abandonar o trabalho) e problemas no trabalho (absenteísmo e licença médica, alta rotatividade, baixo desempenho e má qualidade dos serviços).

O burnout tem sido alvo de interesse para pesquisadores e profissionais de diversas disciplinas. Apesar do volume de 
pesquisa e dos recursos empregados pelos psicólogos da saúde ocupacional para o estudo do burnout, aspectos nevrálgicos para a compreensão desse fenômeno, tais como a sua mensuração, ainda continuam sem solução (Cox, Tisserand, \& Taris, 2005).

Gil-Monte e Peiró (1997) e Schaufeli e Enzmann (1998) descrevem três instrumentos freqüentemente utilizados para avaliar o burnout: o Staff Burnout Scale for Health Professionals (SBS-HP) de Jones (1980); o Burnout Measure (BM) de Pines e Aronson (1988) e o Maslach Burnout Inventory (MBI) de Maslach e Jackson (1986).

O SBS-HP de Jones (1980) é um questionário com 30 itens acompanhados por uma escala de concordância de 6 pontos tipo likert. Desses itens, 20 avaliam a síndrome de burnout e 10 correspondem a uma escala de sinceridade. A análise da estrutura fatorial do SBS-HP indicou a existência dos fatores: Insatisfação Laboral (7 itens; exemplo: acho deprimente o meu ambiente de trabalho); Tensão Psicológica e Interpessoal (7 itens; exemplo: tento evitar o meu supervisor); Doença e Tensão (3 itens; exemplo: sinto-me fadigado no meu trabalho); e Falta de Relações Profissionais com os Pacientes (3 itens; exemplo: no meu trabalho evito o trato com os pacientes). Como esses fatores, no entanto, apresentaram altas correlações entre si $(r$ $=0,59$ a $r=0,82$ ), o conjunto total da escala é interpretado como representando um único fator representativo dos aspectos cognitivos, afetivos, comportamentais e psicofisiológicos da síndrome. Embora a SBS-HP tenha revelado valores adequados de consistência interna $(0,83$ a 0,93$)$, nos estudos de Jones (1980), Grigsby e Mc Knewn (1988) e Tofp e Dillon (1988), a sua unifatorabilidade a torna ampla demais, tendo em vista a inclusão de aspectos generalizáveis a outros fenômenos tais como a insatisfação laboral.

O Burnout Measure (BM) de Pines e Aronson (1988) também é um instrumento unifatorial constituído por 21 itens acompanhados de uma escala de resposta de sete pontos (variando de nunca até sempre), que avalia a freqüência com que o indivíduo apresenta uma série de reações. Esses itens estão distribuídos equitativamente nas seguintes escalas: Esgotamento Físico (7 itens; exemplo: sinto-me sem forças), Esgotamento Emocional (7 itens; exemplo: me sinto deprimido) e Esgotamento Mental (7 itens; exemplo: sinto-me rejeitado). Os escores dessas subescalas são combinados em uma única pontuação que informa o grau de burnout em que a pessoa se encontra. $\mathrm{O}$ BM obteve valores adequados de consistência interna $(0,89$ a 0,94) nos estudos de Mc Cranie, Lambert e Lambert Jr. (1987), Etzion (1984) e Etzion e Pines (1986). No entanto, análises críticas (Schaufeli \& Enzmann, 1998) salientam que esses valores não surpreendem, tendo em vista que vários dos itens da escala podem ser considerados sinônimos ou antônimos. Outra crítica, provavelmente a mais relevante, é a simplificação do fenômeno do burnout retratada no Burnout Measure. A síndrome é reduzida a um simples esgotamento, deixando de lado os seus componentes atitudinais ressaltados em outras abordagens.

O terceiro instrumento, o Inventário de Burnout de Maslach (Maslach Burnout Inventory - MBI) é sem dúvida a medida mais utilizada para a mensuração da síndrome, independentemente das características ocupacionais e da origem da amostra. É consenso na literatura (Gil-Monte \& Peiró, 1997) que o MBI, com os fatores Exaustão Emocional, Despersonalização e Realização Pessoal, estabeleceu a definição operacional padrão da síndrome de burnout. A Exaustão Emocional refere-se a sentimentos de fadiga propiciadores do esgotamento energético emocional. $\mathrm{O}$ fator Despersonalização denota a presença de atitudes negativas do profissional no relacionamento com os usuários dos seus serviços (insensibilidade, indiferença, falta de preocupação, etc.). O fator Realização Pessoal (escala invertida) reflete a deterioração da auto-competência e a falta de satisfação do indivíduo com o seu desempenho e no trabalho.

O MBI é um instrumento multifatorial com três versões. Duas versões são formadas por 22 itens cada, distribuídos entre os fatores Exaustão Emocional (9 itens; p.ex., sinto-me esgotado ao final de um dia de trabalho), Despersonalização (5 itens; p.ex., trato alguns pacientes como se eles fossem objetos) e Realização Pessoal ( 8 itens; p.ex., eu tenho realizado muitas coisas importantes neste trabalho). Cada item está acompanhado por uma escala de resposta de 7 pontos (nunca até todos os dias) que mensura a freqüência de sentimentos relacionados à síndrome. Uma dessas versões destina-se aos profissionais cujo trabalho tem caráter assistencial (MBI - Human Services Survey) e a outra se direciona aos trabalhadores do ensino (MBI - Educators Survey). Uma terceira versão do MBI, a General Survey, com 16 itens, é utilizada em categorias profissionais que não se enquadram nos dois grupos anteriores (Maslach, Jackson, \& Leiter, 1996).

Vários estudos relatam valores adequados de consistência interna $(0,70$ a 0,90$)$ para cada fator do MBI (Goleimbiewsky, Munzenrider, Scherb, \& Billingsley, 1992; Iwanicki \& Schab, 1981; Maslach \& Jackson, 1986). De um modo geral, o fator Exaustão Emocional sempre se apresenta como o fator mais consistente e Despersonalização como o fator menos consistente. Segundo Schaufeli e Enzmann (1998), ocasionalmente têm sido encontrados valores abaixo de 0,70 para o fator Despersonalização. Isso pode ser verificado, por exemplo, no estudo de Gil-Monte (2005a), realizado com diversos profissionais dos setores de saúde, educação e segurança.

Além das medidas mencionadas, muitos outros instrumentos para avaliar a síndrome de burnout têm sido desenvolvidos em diferentes países. Algumas dessas medidas são o Cuestionário de Burnout del Profesorado (CBP) e o Cuestionário Breve de Burnout (CBB), construídos na Espanha por Moreno-Jiménez, Oliver e Aragoneses (1993) e por Moreno-Jiménez, Rodriguez, Alvarez e Caballero (1997), respectivamente; o Copenhagen Burnout Inventory (CBI), desenvolvido por Kristensen, Borritz, Villadsen e Christensen (2005) na Dinamarca, o Oldenburg Burnout Inventory (OLBI), construído por Halsbesleben e Demerouti (2005) na Holanda; o Shirom-Melamed Burnout Measure (SMBM) desenvolvido em Israel por Shirom e Melamed (2006).

Alguns dos instrumentos citados utilizaram como referência o MBI de Maslach e colaboradores. Outros foram desenvolvidos como propostas alternativas, tal como o Copenhagen Burnout Inventory $(\mathrm{CBI})$, que surgiu em discordância ao conceito e a estrutura do burnout apresentadas no MBI (Kristensen et al., 
2005). Os 19 itens do CBI representam os fatores Burnout Pessoal (Alfa $=0,87)$, Burnout Relacionado ao Trabalho (Alfa $=$ 0,87 ) e Burnout Relacionado à Clientela (Alfa $=0,85$ ). Os autores do CBI consideram que a despersonalização e a diminuição da realização pessoal, apesar de serem muito importantes, são fenômenos que não são parte dessa síndrome. Ao invés de componentes de uma síndrome que surge como reação a vivência de um estresse extremo e crônico, a despersonalização pode ser compreendida como uma estratégia de coping, e a diminuição da realização pessoal como uma conseqüência.

No Brasil, foram várias as tentativas de traduzir, adaptar e validar o MBI. Além da investigação da plausibilidade de uma versão brasileira dessa medida, uma preocupação geral foi com a superação das suas limitações relativas às baixas consistências internas dos fatores Despersonalização e Realização Pessoal. Neste sentido, as pesquisas de Lautert (1997), Tamayo (1997, 2003) e Carlotto e Câmara (2004), por exemplo, também desenvolvidas com trabalhadores da educação, saúde e segurança, apresentaram diferentes graus de sucesso no desenvolvimento de versões brasileiras do MBI.

Lautert (1997) desenvolveu uma versão do MBI junto a uma amostra de 341 enfermeiras de dois hospitais universitários. Esse instrumento apresenta os seguintes fatores: Desgaste Emocional (Alfa $=0,86)$, Despersonalização $($ Alfa $=0,69)$ e Incompetência (Alfa $=0,76)$. Pode se observar que Lautert mudou a denominação de dois dos fatores do MBI: Desgaste Emocional em lugar de Exaustão Emocional e Incompetência em vez de Realização Pessoal (escala invertida).

Tamayo $(1997,2003)$ em estudos com amostras de 229 enfermeiras e 290 profissionais da saúde, respectivamente, conseguiu replicar a estrutura tri-fatorial do MBI, mas com índices relativamente baixos quanto à consistência interna para dois dos fatores: 0,69 e 0,73 para Despersonalização e 0,67 e 0,76 para Realização Pessoal. O fator Exaustão Emocional apresentou a mesma tendência observada na maioria das investigações com o MBI e seus coeficientes Alfa foram 0,82 e 0,84.

Finalmente, Carlotto e Câmara (2004), com uma amostra de 563 professores, também verificaram a mesma estrutura tri-fatorial. Mas, apesar dos fatores Exaustão Emocional e Realização Pessoal terem melhorado a sua consistência interna (Alfa $=0,88$ e 0,82 , respectivamente), o fator Despersonalização apresentou um índice muito baixo (Alfa $=0,58)$.

Tanto nos estudos brasileiros quanto em validações do MBI desenvolvidas em outros países, verificou-se a importância, em termos conceituais e de consistência interna, do fator Exaustão Emocional. Os outros dois fatores, além da disputa conceitual (Kristensen et al., 2005) também apresentam problemas de confiabilidade.

Além dos estudos de replicação e adaptação de medidas como o MBI, faltam trabalhos que procurem desenvolver novos instrumentos de mensuração da síndrome de burnout baseados na cultura brasileira. Para além das questões psicométricas, é necessário que existam investigações das possíveis estruturas fatoriais também no nosso meio cultural e organizacional. Análises exploratórias da possível estrutura fatorial de qualquer instrumento não podem revelar fatores que não tenham sido expressos por dois ou mais itens. Isto é, não é possível identificar uma estrutura fatorial se não forem elaborados meios possíveis de expressão desses fatores em primeiro lugar. Quando o ponto de partida se constitui de itens representativos de estruturas fatoriais encontradas em outras culturas, os resultados estarão limitados ou a uma replicação (o que já é bastante promissor) ou então a uma mera reordenação, muitas vezes sem sentido, da estrutura fatorial representada originalmente pelos itens. Em outras palavras, a simples replicação ou adaptação limita severamente a possibilidade de descoberta de novos fatores ou de novos reordenamentos significativos.

Nessa perspectiva, este trabalho relata dois estudos os quais procuram desenvolver uma nova medida da síndrome de burnout, a Escala de Caracterização do Burnout (ECB), possibilitando (1) uma melhor investigação da generalização da estrutura tri-fatorial do MBI na cultura brasileira bem como a presença de novos fatores, e (2) que apresente melhores qualidades psicométricas do que as medidas utilizadas no Brasil.

\section{Estudo 1}

O Estudo 1 consistiu na construção de uma primeira versão longa e validação fatorial preliminar de um novo instrumento de mensuração da síndrome denominado Escala de Caracterização do Burnout (ECB). Para assegurar maior representatividade e consistência da estrutura tri-fatorial do MBI, bem como de possíveis novos fatores, foram utilizados itens de validações anteriores do MBI (Tamayo, 1997, 2003), itens retirados do Staff Burnout Scale for Health Professionals (Jones, 1980), Burnout Measure (Pines \& Aronson, 1988) e Cuestionario Breve de Burnout (Moreno-Jiménez et al., 1997) e, ao mesmo tempo, também foram acrescentados novos itens elaborados pelos autores após entrevistas realizadas com trabalhadores das áreas de saúde e educação.

A primeira versão da ECB ficou constituída por 77 (setenta e sete) itens. Para verificar a clareza e a compreensão desses itens foi realizada uma análise semântica com trabalhadores do estrato mais baixo de habilidade da população alvo ( 8 auxiliares de enfermagem com segundo grau completo de escolaridade) e com trabalhadores do estrato mais alto de habilidade (3 enfermeiras e 1 professora de escola). $O$ processo de validação semântica foi realizado em duplas e individualmente. Esse processo resultou em mudanças na redação de vários itens e na retirada de seis itens considerados confusos ou ambíguos.

Em seguida, um júri composto por quatro professores com interesse acadêmico em estresse ocupacional e síndrome de burnout e por quatro trabalhadores da população em que $\mathrm{o}$ instrumento seria validado, classificou cada item dentro de uma estrutura fatorial sugerida pelos autores. Foi obtida uma concordância superior a $60 \%$ na classificação de 30 dos 71 itens. Provavelmente, devido à relação existente entre os fatores, um alto número de itens foi classificado em mais de um fator simultaneamente. Entretanto, esses itens foram mantidos para observar suas características psicométricas no processo de validação estatística do instrumento.

AECB foi concebida para uso junto a diferentes profissionais (profissionais de saúde, professores, policiais, terapeutas, entre outros). 


\section{Método}

\section{Participantes e Procedimentos}

Uma amostra de 373 trabalhadores de ambos os sexos (274 mulheres, 93 homens, 6 pessoas não informaram o gênero), com idade média de 34 anos $(D P=8,36)$ respondeu a primeira versão da Escala de Caracterização do Burnout (ECB). Esta primeira versão consistiu de um questionário de auto-relato composto de 71 itens acompanhados por uma escala tipo likert de 5 pontos $(1=$ Nunca e $5=$ Sempre $)$ juntamente com questões sobre características sócio-demográficas. Esses trabalhadores ocupavam o cargo de auxiliar ou técnico de enfermagem $(n=$ 172 ), enfermeiros lotados em diferentes especialidades clínicas $(n=46)$ e professores de primeiro e segundo grau $(n=155)$.

A escala foi aplicada individualmente e em grupo em hospitais e escolas públicas e particulares do Distrito Federal.

\section{Resultados}

As respostas aos 71 itens da ECB foram submetidas inicialmente a uma análise dos componentes principais (principal components) para estimar o número de fatores, a presença de valores extremos, a ausência de multicolinearidade e a fatorabilidade da matriz de correlação. As respostas faltosas foram desconsideradas da análise. Vinte dos 373 participantes foram identificados como casos extremos multivariados de acordo com o procedimento sugerido por Tabachnick e Fidell (1996). Optou-se, no entanto, por não retirar esses casos, considerando que a sua existência estava mais relacionada à presença de um subgrupo de participantes com índices diferenciados na ECB, do que a casos extremos atípicos.

O gráfico scree plot sugeriu a presença de 2 a 4 componentes. O critério de Harman, acerca da importância dos fatores, revelou que até a extração de 4 componentes obtinha-se um acréscimo de $3 \%$ na variância total explicada. Esses quatro componentes apresentaram eigenvalue maior que 2,0, explicando $42 \%$ da variância total. Os índices $K M O=0,93$ e o qui-quadrado do Teste de Esfericidade de Bartlett estatisticamente significativo sugeriram a adequação da matriz para ser submetida à análise fatorial (Pasquali, 1998).

Uma vez verificada a adequação da matriz foram realizadas três análises fatoriais com o método dos eixos principais (principal axis factoring) com rotação oblíqua (oblimin), para identificar a melhor solução fatorial (2, 3 ou 4 fatores). Tanto em termos das propriedades psicométricas, quanto das qualidades interpretativas, a melhor solução foi a de quatro fatores (ver Tabela 1). Essa solução resultou em um instrumento constituído por 46 itens, com carga superior a 0,40. Vinte e três das 71 afirmativas não apresentaram cargas significativas nos quatro fatores extraídos e dois itens foram eliminados por apresentarem cargas elevadas em mais de um fator. Os itens com cargas inferiores a 0,40 foram omitidos da Tabela 1 para facilitar a visualização. $\mathrm{O}$ índice de confiabilidade e número de itens de cada fator foram: Exaustão Emocional (20 itens, Alfa $=0,93$ ), Desumanização (12 itens, Alfa =0,88), Realização Pessoal (6 itens, Alfa $=0,77)$ e Decepção no Trabalho ( 8 itens,
Alfa $=0,80)$.

Um exame dos itens constituintes de cada fator, listados na Tabela 1, mostra que os itens do primeiro fator, Exaustão Emocional, transmitem a idéia de esgotamento, cansaço e desgaste no trabalho. O segundo fator, Desumanização, está representado por itens que sugerem dureza emocional, desinteresse e atitudes negativas no trato com os clientes. $\mathrm{O}$ terceiro fator, Realização Pessoal, apresenta itens cujo conteúdo denota satisfação, compromisso e confiança no desempenho no trabalho. Por último, o quarto fator, Decepção no Trabalho, reúne itens sobre a desesperança com respeito ao progresso profissional, a insatisfação e falta de compromisso no trabalho e a perda da confiança na própria capacidade para realizá-lo adequadamente. Estes dois últimos fatores representam, de fato, apenas versões positivas e negativas do fator Diminuição da Realização Pessoal do MBI de Maslach e Jackson (1986). A correlação moderada e negativa entre eles $(r=-0,35)$ bem como o conteúdo dos seus itens sugere claramente que se trata de um único fator.

\section{Estudo 2}

A estrutura fatorial resultante do Estudo 1 apresentou os mesmos fatores encontrados na estrutura tri-fatorial do MBI, mas com a novidade de um quarto fator, que se revelou, junto com o terceiro (vide Tabela 1), uma divisão do fator Realização Pessoal do MBI.

O surgimento de dois novos fatores resultantes da divisão do fator Realização Pessoal tem ocorrido em outros estudos. $\mathrm{Na}$ pesquisa de Gil-Monte (2005a), por exemplo, foram identificados dois fatores relacionados à realização pessoal (Self-Competence e Existencial Component), mas como também apresentaram alta correlação $(r=0,88)$, foi decidido manter a estrutura tri-fatorial original do MBI (Exaustão Emocional, Despersonalização e Realização Pessoal).

De forma semelhante, o Estudo 1 também revelou dois fatores constituídos por itens elaborados para representar o fator Realização Pessoal. É possível que a diferenciação em dois fatores seja uma conseqüência de aspectos semânticos e não de diferenças conceituais. Itens com afirmações positivas tendem a eliciar respostas comuns com outros itens semelhantes e não com as respostas dadas a itens escritos na forma negativa, mesmo quando os dois conjuntos de itens estejam relacionados ao mesmo assunto.

Portanto, para investigar a possibilidade da existência de um novo fator, que poderia surgir da divisão do fator Realização Pessoal, bem como para testar a estabilidade dos fatores Exaustão Emocional e Desumanização, foi realizada uma nova aplicação da ECB. Tratando-se de outra amostra, e na presença de resultados ambíguos do Estudo 1, quanto ao possível número de fatores conceitualmente relevantes, considerou-se necessário o recurso a uma nova análise fatorial exploratória. No atual estágio do desenvolvimento da ECB, o recurso a uma análise fatorial confirmatória seria prematuro uma vez que ainda não é possível a proposição de estruturas fatoriais que representem claramente alternativas excludentes ao instrumento ECB.

Participantes e Procedimentos 
Tabela 1

Carga fatorial, comunalidades $\left(h^{2}\right)$, eigenvalues e percentual de variância da análise fatorial da Escala de Caracterização do Burnout (ECB) no Estudo 1.

\begin{tabular}{|c|c|c|c|c|c|}
\hline \multirow{2}{*}{ Itens } & \multicolumn{4}{|c|}{ Fatores } & \multirow{2}{*}{$h^{2}$} \\
\hline & $\mathrm{I}$ & II & III & IV & \\
\hline Sinto-me esgotado ao final de um dia de trabalho & 0,75 & & & & 0,62 \\
\hline Eu me sinto desgastado com o meu trabalho & 0,74 & & & & 0,69 \\
\hline Meu trabalho me faz sentir emocionalmente exausto & 0,70 & & & & 0,65 \\
\hline Eu me sinto sugado pelo meu trabalho & 0,62 & & & & 0,63 \\
\hline Acho que estou trabalhando demais no meu emprego & 0,62 & & & & 0,64 \\
\hline Sinto que este trabalho está me destroçando & 0,59 & & & & 0,66 \\
\hline $\begin{array}{l}\text { Quando me levanto de manhã, sinto cansaço só de pensar que tenho } \\
\text { que encarar mais um dia de trabalho }\end{array}$ & 0,59 & & & & 0,64 \\
\hline Eu me sinto saturado com meu trabalho & 0,57 & & & & 0,71 \\
\hline Meu trabalho afeta negativamente meu bem estar psicológico & 0,55 & & & & 0,69 \\
\hline Eu me sinto desanimado com meu trabalho & 0,55 & & & & 0,66 \\
\hline Eu me sinto desiludido com meu trabalho & 0,52 & & & & 0,64 \\
\hline Meu trabalho me exige mais do que posso dar & 0,51 & & & & 0,67 \\
\hline Eu me sinto deprimido com meu trabalho & 0,50 & & & & 0,68 \\
\hline Eu me sinto frustrado com meu trabalho & 0,50 & & & & 0,65 \\
\hline Trabalhar direitamente com pessoas me deixa muito estressado & 0,50 & & & & 0,70 \\
\hline Sinto-me infeliz com meu trabalho & 0,48 & & & & 0,61 \\
\hline Meu trabalho afeta negativamente minha saúde física & 0,48 & & & & 0,60 \\
\hline $\begin{array}{l}\text { Sinto que a carga emocional do meu trabalho é superior àquela que } \\
\text { posso suportar }\end{array}$ & 0,47 & & & & 0,60 \\
\hline $\begin{array}{l}\text { Meu trabalho me faz sentir como se estivesse no limite das minhas } \\
\text { possibilidades }\end{array}$ & 0,45 & & & & 0,55 \\
\hline Sinto-me emocionalmente vazio com meu trabalho & 0,40 & & & & 0,63 \\
\hline Perco a paciência com alguns pacientes & & $-0,60$ & & & 0,67 \\
\hline Eu me sinto rejeitado por alguns pacientes & & $-0,57$ & & & 0,59 \\
\hline Trato alguns pacientes com indiferença, quase de forma mecânica & & $-0,57$ & & & 0,72 \\
\hline Sinto que desagrado a alguns pacientes & & $-0,56$ & & & 0,60 \\
\hline Enfureço-me com alguns pacientes & & $-0,55$ & & & 0,52 \\
\hline Fico de mal humor quando lido com alguns pacientes & & $-0,54$ & & & 0,59 \\
\hline Trato alguns pacientes com frieza & & $-0,54$ & & & 0,66 \\
\hline Trato alguns pacientes com distanciamento & & $-0,51$ & & & 0,68 \\
\hline Trato alguns pacientes com cinismo & & $-0,48$ & & & 0,59 \\
\hline Sinto que os pacientes me culpam por alguns dos seus problemas & & $-0,48$ & & & 0,61 \\
\hline Sinto que alguns pacientes são "meus inimigos" & & $-0,45$ & & & 0,57 \\
\hline Evito o trato com alguns pacientes & & $-0,45$ & & & .601 \\
\hline Trabalhar em parceria com meus pacientes me estimula & & & 0,64 & & 0,64 \\
\hline Acho que trato adequadamente os problemas dos meus pacientes & & & 0,50 & & 0,71 \\
\hline Compreendo facilmente os sentimentos dos meus pacientes & & & 0,50 & & 0,59 \\
\hline Eu me sinto identificado com meu trabalho & & & 0,48 & & 0,60 \\
\hline Acho que as coisas que realizo no meu trabalho valem a pena & & & 0,45 & & 0,52 \\
\hline Eu me sinto cheio de energia para trabalhar & & & 0,42 & & 0,63 \\
\hline Acho que meu trabalho parece sem sentido & & & & 0,57 & 0,65 \\
\hline Sinto vontade de me isolar no ambiente de trabalho & & & & 0,54 & 0,69 \\
\hline Meu trabalho me faz sentir como se estivesse "num beco sem saída" & & & & 0,49 & 0,67 \\
\hline Sinto-me desesperado com meu trabalho & & & & 0,48 & 0,63 \\
\hline Eu me sinto inútil no meu trabalho & & & & 0,48 & 0,55 \\
\hline Evito o relacionamento com a chefia & & & & 0,47 & 0,60 \\
\hline Evito o relacionamento com meus colegas de trabalho & & & & 0,44 & 0,62 \\
\hline Acho que realizo meu trabalho inadequadamente & & & & 0,41 & 0,68 \\
\hline Eigenvalues & 20,5 & 4,1 & 2,9 & 2,2 & \\
\hline Percentual de variância & 28,9 & 5,8 & 4,1 & 3,1 & \\
\hline Percentual de variância acumulada & 28,9 & 34,8 & 38,9 & 42,0 & \\
\hline Coeficiente Alfa de Cronbach & 0,94 & 0,88 & 0,76 & 0,80 & \\
\hline
\end{tabular}

Fator I - Exaustão Emocional. Fator III - Realização pessoal.

Fator II - Desumanização. $\quad$ Fator IV - Decepção no trabalho. 
A amostra participante neste estudo foi constituída por 787 trabalhadores do setor policial e penitenciário de ambos os sexos (590 homens, 185 mulheres, 12 não informaram o gênero). Esses trabalhadores ocupavam cargo de delegado $(n=52)$, agente de polícia $(n=488)$, escrivão $(n=93)$ e agente penitenciário $(n=$ $153)$. A idade média dos respondentes foi 35,32 anos $(D P=5,20)$. O tempo de serviço foi de 9,14 anos $(D P=6,59)$.

A escala foi aplicada em todas as delegacias especializadas e circunscricionais de um órgão de segurança pública e alguns presídios do Distrito Federal. A aplicação foi feita de forma individual e grupal sendo disponibilizadas urnas para o depósito dos questionários preenchidos.

\section{Resultados}

Da mesma forma que no Estudo 1, foram realizadas análises exploratórias para identificar erros de digitação, respostas faltosas, casos extremos e para determinar os pressupostos exigidos para as análises multivariadas (Tabachnick \& Fidell, 1996). Não foram identificados respostas extremas univariadas nem foi necessário efetuar nenhuma transformação das variáveis.

Inicialmente, uma análise dos componentes principais indicou a provável existência de 3 a 4 componentes (gráfico scree plot), revelou um índice $K M O$ igual a 0,96 e um coeficiente quiquadrado no Teste de Esfericidade de Bartlett estatisticamente significativo, comprovando a fatoriabilidade da matriz de correlações. Os componentes apresentaram eigenvalue superior a 2,0, explicando $45 \%$ da variância total e respeitando o critério de Harman de acréscimo de 3\% da variância total explicada. Em seguida, foram rodadas duas análises fatoriais com o método de extração dos eixos principais (principal axis factoring) e rotação oblíqua (oblimin), para determinar a melhor solução fatorial apresentada na Tabela 2 .

Pode-se observar na Tabela 2 que a solução mais adequada foi a de três fatores constituídos por 35 itens com carga superior a 0,45 (no Estudo 2, optou-se por utilizar um ponto de corte mais alto da carga fatorial com a finalidade de diminuir o número de itens do instrumento). A análise fatorial identificou os seguintes fatores: Exaustão Emocional (12 itens, Alfa = 0,93), Desumanização (10 itens, Alfa $=0,84)$ e Decepção no Trabalho (13 itens, Alfa $=0,90)$.

\section{Discussão}

O objetivo central deste trabalho foi a construção e validação fatorial de um instrumento brasileiro para mensuração da síndrome de burnout. Seguindo a tendência da escala mais conhecida nas investigações sobre a síndrome, os dois estudos apresentados apontaram evidências de uma estrutura tri-fatorial na Escala de Caracterização do Burnout (ECB).

A estrutura de quatro fatores encontrada no primeiro estudo pode ser considerada como o resultado de um artifício semântico devido à inclusão na escala de itens positivos e negativos. Isto foi comprovado no Estudo 2 quando apenas três itens positivos ( $E u$ me sinto identificado com meu trabalho; As coisas que realizo no meu trabalho valem a pena; Eu me sinto cheio de energia para trabalhar) permaneceram no terceiro fator da estrutura fatorial tradicional (fator Decepção no Trabalho). Esses itens poderiam até ser suprimidos; procedimento esse que não afetaria o índice de consistência interna do terceiro fator (iria de 0,90 para 0,89 ), nem o seu significado.

Gil-Monte (2005b), ao tratar dos problemas psicométricos do MBI, aponta que estudos têm concluído que resultados obtidos mediante a apresentação de itens positivos para avaliar baixa realização pessoal, quando comparados aos resultados obtidos com a expressão negativa desses itens, afetam significativamente diferentes parâmetros estatísticos. Por essa razão, esse autor recomenda reelaborar a escala de "realização pessoal" para apresentar os itens de forma negativa.

Outros autores, como Bresó, Salanova e Schaufeli (2007), também criticam a forma como é mensurada a baixa realização pessoal, no Inventário de Burnout de Maslach. Nesse instrumento, a avaliação dessa dimensão do burnout é realizada mediante itens positivos (que sugerem realização pessoal). Posteriormente, esses itens são invertidos para transformá-los em um indicador da síndrome (a baixa realização pessoal).

De acordo com Bresó et al. (2007), a adoção desse procedimento pressupõe que altos escores em realização pessoal são equivalentes a escores inferiores em baixa realização pessoal e vice-versa. Entretanto, o pressuposto que considera a realização pessoal e a baixa realização pessoal como pólos perfeitamente opostos de uma mesma dimensão, pode ser contestado. Em conseqüência, estudos desenvolvidos recentemente para explorar esses aspectos (Bresó et al., 2007; Schaufeli \& Salanova, 2007) recomendam que o componente do burnout "baixa realização pessoal" seja mensurado por meio de uma escala de itens negativos que retratem esse tipo de conteúdo, em lugar de usar uma escala com afirmativas positivas de realização pessoal, que posteriormente deva ser invertida para poder avaliar a falta de realização pessoal. Nessas pesquisas foram encontradas correlações mais altas dos fatores Exaustão Emocional e Despersonalização com a Baixa Realização Pessoal, quando este último fator foi mensurado com afirmativas negativas e não com itens positivos invertidos posteriormente para fins da análise.

O mesmo tipo de evidência foi encontrada no Estudo 1 com correlações mais altas entre o fator Decepção no Trabalho (itens negativos) e as dimensões Exaustão Emocional e Despersonalização do que as correlações apresentadas pelo fator Realização Pessoal (itens positivos) com essas dimensões.

Novamente, o fator exaustão emocional revelou-se como o fator mais consistente também na ECB. Esse mesmo resultado foi verificado na versão original do Inventário de Burnout de Maslach (Maslach \& Jackson, 1986), nas versões Brasileiras deste inventário (Carlotto \& Câmara, 2004; Lautert, 1997; Tamayo 1997, 2003) e no Cuestionario Breve de Burnout (Moreno-Jiménez et al., 1997). A consistência interna do fator exaustão emocional tem sido, reiteradamente, mais alta do que a verificada para os outros dois fatores da síndrome de burnout.

É provável que isso aconteça, porque a dimensão da síndrome de burnout, exaustão emocional, relaciona-se diretamente com aspectos do estresse individual. A exaustão emocional parece ser a manifestação mais representativa da síndrome e as pessoas descrevem a sua experiência com ela a 
Tabela 2

Carga fatorial, comunalidades $\left(h^{2}\right)$, eigenvalues e percentual de variância da análise fatorial da Escala de Caracterização do Burnout (ECB) no Estudo 2.

\begin{tabular}{|c|c|c|c|c|}
\hline \multirow{2}{*}{ Itens } & \multicolumn{3}{|c|}{ Fatores } & \multirow{2}{*}{$h^{2}$} \\
\hline & I & II & III & \\
\hline Eu me sinto desgastado com meu trabalho & 0,73 & & & 0,73 \\
\hline Eu me sinto sugado pelo meu trabalho & 0,72 & & & 0,69 \\
\hline $\begin{array}{l}\text { Meu trabalho me faz sentir como se estivesse no limite das minhas } \\
\text { possibilidades }\end{array}$ & 0,66 & & & 0,64 \\
\hline Meu trabalho me exige mais do que posso dar & 0,65 & & & 0,58 \\
\hline Meu trabalho me faz sentir emocionalmente exausto & 0,65 & & & 0,68 \\
\hline Meu trabalho afeta negativamente minha saúde física & 0,64 & & & 0,55 \\
\hline Sinto-me esgotado ao final de um dia de trabalho & 0,63 & & & 0,58 \\
\hline $\begin{array}{l}\text { Sinto que a carga emocional do meu trabalho é superior àquela que } \\
\text { posso suportar }\end{array}$ & 0,59 & & & 0,53 \\
\hline Acho que estou trabalhando demais no meu emprego & 0,58 & & & 0,58 \\
\hline Meu trabalho afeta negativamente meu bem-estar psicológico & 0,57 & & & 0,59 \\
\hline Sinto que meu trabalho está me destroçando & 0,56 & & & 0,68 \\
\hline Eu me sinto saturado com meu trabalho & 0,54 & & & 0,68 \\
\hline Trato algumas vítimas com frieza & & 0,73 & & 0,65 \\
\hline Perco a paciência com algumas vítimas & & 0,67 & & 0,67 \\
\hline Fico de mau humor quando lido com algumas vítimas & & 0,62 & & 0,62 \\
\hline Enfureço-me com algumas vítimas & & 0,61 & & 0,63 \\
\hline Trato algumas vítimas com indiferença, quase de forma mecânica & & 0,60 & & 0,68 \\
\hline Trato algumas vítimas com distanciamento & & 0,59 & & 0,67 \\
\hline Sinto que algumas vítimas são "meus inimigos" & & 0,54 & & 0,46 \\
\hline Sinto que desagrado algumas vítimas & & 0,51 & & 0,56 \\
\hline Trato algumas vítimas com cinismo & & 0,50 & & 0,50 \\
\hline Evito o trato com algumas vítimas & & 0,49 & & 0,47 \\
\hline Sinto-me emocionalmente vazio com meu trabalho & & & $-0,70$ & 0,66 \\
\hline Eu me sinto desiludido com meu trabalho & & & $-0,66$ & 0,67 \\
\hline Eu me sinto identificado com meu trabalho & & & 0,62 & 0,56 \\
\hline Acho que meu trabalho parece sem sentido & & & $-0,61$ & 0,49 \\
\hline Eu me sinto desanimado com meu trabalho & & & $-0,60$ & 0,71 \\
\hline Acho que as coisas que realizo no meu trabalho valem a pena & & & 0,59 & 0,59 \\
\hline Eu me sinto frustrado com meu trabalho & & & $-0,57$ & 0,51 \\
\hline Meu trabalho me faz sentir como se estivesse num beco sem saída & & & $-0,55$ & 0,53 \\
\hline Sinto-me desesperado com meu trabalho & & & $-0,54$ & 0.48 \\
\hline Eu me sinto inútil no meu trabalho & & & $-0,52$ & 0,39 \\
\hline $\begin{array}{l}\text { Quando me levanto de manhã sinto cansaço só de pensar que tenho } \\
\text { que encarar mais um dia de trabalho }\end{array}$ & & & $-0,49$ & 0,54 \\
\hline Eu me sinto cheio de energia para trabalhar & & & 0,48 & 0,39 \\
\hline Sinto-me infeliz com meu trabalho & & & $-0,47$ & 0,44 \\
\hline Eigenvalues & 14,6 & 3,9 & 2,19 & \\
\hline Percentual de variância & 31,7 & 8,6 & 4,7 & \\
\hline Percentual de variância acumulada & 31,7 & 40,4 & 45,1 & \\
\hline Coeficiente Alfa de Cronbach & 0,93 & 0,84 & 0,90 & \\
\hline
\end{tabular}

Fator I - Exaustão Emocional. Fator II - Desumanização. Fator III - Decepção no trabalho.

partir dos seus sentimentos de exaustão. Provavelmente, por essa razão, entre os três aspectos da síndrome, a exaustão é o mais amplamente reportado e o mais profundamente analisado (Cordes \& Dougherty, 1993; Maslach, 1993; Maslach, Schaufeli \& Leiter, 2001).

Talvez seja mais fácil para as pessoas reconhecer os sentimentos de desgaste provocados pelo trabalho do que atitudes de descaso e indiferença no relacionamento com os clientes, como acontece no caso da despersonalização ou desumanização. Também é mais fácil admitir o cansaço e o esgotamento produzidos pelo trabalho do que os sentimentos de inadequação, ineficácia e desesperança decorrentes da falta de realização pessoal e decepção no trabalho.
Nesse sentido, embora vários itens das dimensões Exaustão Emocional e Realização Pessoal do Inventário de Burnout de Maslach (1986) tenham permanecido na análise fatorial da ECB, apenas um item do fator Despersonalização permaneceu. Provavelmente os outros não se mantiveram porque o seu conteúdo confronta, de maneira muito forte e direta, a imagem e o papel social do profissional.

Alguns itens do fator Despersonalização do MBI que tratam da insensibilidade do indivíduo no relacionamento com os clientes, dificilmente são respondidos com sinceridade devido à influência da desejabilidade social (Tamayo, 1997). Aparentemente, as afirmativas do fator Desumanização da ECB sofreram um menor impacto da desejabilidade social. No entanto, 
novos estudos devem ser desenvolvidos para elucidar esse aspecto. Além disso, o fator Desumanização da ECB evidenciou um índice de consistência interna superior ao apresentado pelo fator Despersonalização das versões Brasileiras do Inventário de Burnout de Maslach, MBI (Carlotto \& Câmara, 2004; Lautert, 1997; Tamayo, 1997, 2003). É provável que esse resultado tenha decorrido do maior número de itens utilizado para aferir esse fator.

A diferença entre o Burnout Measure (BM) e a Escala de Caracterização do Burnout (ECB) encontra-se no fato de que a primeira medida avalia a síndrome de burnout de forma unifatorial, reduzindo o fenômeno a um simples esgotamento (Enzmann, Schaufeli, Janssen, \& Rozeman, 1998). Por essa razão, esse instrumento não consegue captar os aspectos atitudinais e de auto-avaliação presentes na síndrome, assemelhando-se mais a uma medida ortodoxa de estresse. Não é recomendável desenvolver um instrumento unifatorial a partir de um construto multidimensional. Além disso, o Burnout Measure foi desenvolvido para avaliar essa síndrome em um contexto geral, razão pela qual nenhum dos seus itens faz referência ao trabalho.

Outra medida unifatorial, a Staff Burnout Scale for Health Professionals (SBS-HP), avalia a síndrome utilizando variáveis muito amplas, inespecíficas e comuns a outros fenômenos, tais como a insatisfação laboral e os problemas de saúde. Ao contrário da SBS-HP, a ECB trabalha com uma perspectiva multifatorial que lhe permite considerar aspectos mais específicos e delimitar o fenômeno com maior precisão.

A principal vantagem da $\mathrm{ECB}$ em relação ao Cuestionário Breve de Burnout (CBB) são as suas propriedades psicométricas. Os baixos índices de confiabilidade do CBB podem decorrer do número reduzido de itens para cada fator (três itens por fator). A ECB apresenta um maior número de itens por fator e índices de consistência muito superiores aos alcançados pelo CBB. Além disso, o CBB também apresenta itens que, por transmitir mais de uma idéia, são potencialmente ambíguos. Finalmente, a ECB apresenta propriedades psicométricas superiores as outras versões brasileiras do Inventário de Burnout Maslach.

\section{Referências}

Bresó, E., Salanova, M., \& Schaufeli, W. B. (2007). In search of the "third dimension" of burnout: efficacy or inefficacy? Applied Psychology: an International Review, 56 (3), 460-478.

Brill, P. L. (1984). The need for an operational definition of burnout. Family and Community Health, 6, 12-24.

Byrne, B. (1993a). Burnout: testing for the validity, replication and invariance of causal structure across elementary, intermediate and secondary teachers. American Educational Research Journal, 31(3), 645-673.

Byrne, B. (1993b). The Maslach Burnout Inventory: testing for factorial validity and invariance across elementary, intermediate and secondary teachers. Journal of_Occupational and Organizational Psychology, 66, 197-212.

Carlotto, M. S., \& Câmara, S. G. (2004). Análise fatorial do Maslach Burnout Inventory (MBI) em uma amostra de professores de instituições particulares. Psicologia em Estudo, 9(3), 499-505.

Cordes, C. L., \& Dougherty, T. W. (1993). A review and integration and research on job burnout. Academy of Management Review, 18, 621-656.

Cox, T., Tisserand, M., \& Taris, T. (2005). The conceptualization and measurement of burnout: Questions and directions. Work \& Stress, 19(3), 187-191.

Craig, G. J. (1986). Human development. Englewood Cliffs: Prentice-Hall.

Edelwich, J., \& Brodsky, A. (1980). Burn-out: stages of disillusionment in the helping professions. New York: Human Sciences Press.

Enzmann, D., Schaufeli, W. B., Janssen, P., \& Rozeman, A. (1998). Dimensionality and validity of the Burnout Measure. Journal of Occupational and Organizational Psychology, 71, 331-351.

Etzion, D. (1984). Moderating effect of social support on the stress-burnout relationship. Journal of Applied Psychology, 69(4), 615-622.

Etzion, D., \& Pines, A. (1986). Sex and culture in burnout and coping among human service professionals: a social psychological perspective. Journal of Cross-Cultural Psychology, 17(2), 191-209.

Garden, A. M. (1989). Burnout: the effect of psychological type on research findings. Journal of Occupational Psychology, 62, 223-234.

Garden, A. M. (1991). Relation between burnout and performance. Psychological Reports, 68, 963-977.

Gil-Monte, P. R. (2005a). Factorial validity of the Maslach Burnout Inventory (MBI-HSS) among Spanish professionals. Revista de Saúde Pública, 39(1), $1-8$.

Gil-Monte, P. R. (2005b). El sindrome de quemarse por el trabajo (burnout): una enfermedad laboral en la sociedad del bienestar. Madrid: Ediciones Pirámide.

Gil-Monte, P. R., \& Peiró, J. M. (1997). Desgaste psiquico en el trabajo: el sindrome de quemarse. Madrid: Síntesis.

Golembiewski, R. T., Munzenrider, R. F., Scherb, K., \& Billingsley, W. (1992). Burnout and psychiatric cases: Early evidences of an association. Anxiety, Stress and Coping, 5, 69-78.

Grigsby, D. W., \& Mc Knewn, M. A. (1988). Work-stress burnout among paramedics. Psychological Reports, 63, 55-64.

Halbesleben, J. R. B., \& Demerouti, E. (2005). The construct validity of an alternative measure of burnout: Investigating the English translation of the Oldenburg Burnout Inventory. Work \& Stress, 19(3), 208-220.

Iwanicki, E. F., \& Schwab, R. L. (1981). A cross validation study of the Maslach Burnout Inventory. Educational and Psychological Measurement, 41, 1167-1174.

Jones, J. (1980). The staff burnout scale: a validity study. Trabalho apresentado na $52^{\mathrm{a}}$ Reunião Anual da Midwestern Psychological Association, St Louis, Missouri.

Kristensen, T. S., Borritz, M., Villadsen, E., \& Christensen, K. B. (2005). The Copenhagen Burnout Inventory: A new tool for the assessment of burnout. Work \& Stress, 19(3), 192-207.

Lautert, L. (1997). O desgaste profissional: estudo empírico com enfermeiras que trabalham em hospitais. Revista Gaúcha de Enfermagem, 18(2), 133-144.

Leiter, M. P., \& Meechan, K. A. (1986). Role structure and burnout in the field of human services. The Journal of Applied Behavioral Science, 22, 47-52.

Mallet, K., Jurs, S., Price, J., \& Slenker, S. (1991). Relation among burnout, death, anxiety, and social support in hospice and critical care nurses. Psychological Reports, 68, 1347-1359.

Maslach, C. (1978). The client role in staff burn-out. Journal of Social Issues, 34(4), 111-124

Maslach, C. (1993). Burnout: a multidimensional perspective. In W. Schaufelli, C. Maslach \& T. Marek (Orgs.), Professional burnout: recent developments in theory and research (pp. 19-32). Washington: Taylor \& Francis.

Maslach, C., \& Jackson, S. E. (1986). Maslach Burnout Inventory. Palo Alto: Consulting Psychological Press.

Maslach, C., Jackson, S. E., \& Leiter, M. P. (1996). Maslach Burnout Inventory Manual. Palo Alto: Consulting Psychological Press.

Maslach, C., \& Leiter, M. (1997). The truth about burnout: how organizations cause personal stress and what to do about it. San Francisco: Jossey-Bass Publishers.

Maslach, C., Schaufeli, W. B., \& Leiter, M. P. (2001). The job burnout. Journal 
of Applied Psychology, 52, 397-422.

Mc Cranie, E. W., Lambert, V., \& Lambert Jr., C. (1987). Work stress, hardiness and burnout among hospital staff nurses. Nursing Research, 36(6), 374378.

Meier, S. (1983). Toward a theory of burnout. Human Relations, 36, 899-910.

Meier, S. (1984). The construct validity of burnout. Journal of Occupational Psychology, 57, 211-219.

Moreno-Jiménez, B., Oliver, C., \& Aragoneses, A. (1993). Configuración especifica del estrés laboral asistencial en el profesorado de Educación Media. Manuscrito não publicado. Centro Nacional de Investigación Educativa (CIDE). Madrid.

Moreno-Jiménez, B., Rodriguez, R. B., Alvarez, A. M., \& Caballero, T. M. (1997). La evaluación del burnout. Problemas y alternativas. El CBB como evaluación de los elementos del proceso. Revista de Psicología del Trabajo $y$ de las Organizaciones, 13(2), 185-207.

Pasquali, L. (1998). Análise fatorial: Um manual teórico-prático. Brasília: LabPAM - UnB.

Pines, A., \& Aronson, E. (1988). Career burnout: Causes and cures. New York: Free Press.

Schaufeli, W., \& Enzmann, D. (1998). The burnout companion to study and practice: a critical analysis. London: Taylor \& Francis.

Schaufeli, W. B., \& Salanova, M. (2007). Efficacy or inefficacy, that's the question:
Burnout and work engagement, and their relationship with efficacy beliefs. Anxiety, Stress, and Coping, 20(2), 177-196.

Shiron, A., \& Melamed, S. (2006). A comparison of the construct validity of two burnout measures in two groups of professionals. International Journal of Stress Management, 13(2), 176-200.

Silverstein, R. (1982). How experienced psychotherapist cope with burnout at a state mental hospital. Dissertation prospectus, South Illinois University, Carbondale.

Tabachinick, B., \& Fidell, L. (1996). Using Multivariate Statistics. New York: Harper Collins College Publishers.

Tamayo, M. R. (1997). Relação entre a sindrome do burnout e os valores organizacionais no pessoal de enfermagem de dois hospitais públicos. Dissertação de mestrado não-publicada, Universidade de Brasília, Brasília.

Tamayo, M. R. (2002). Burnout: Relação com a afetividade negativa, o coping no trabalho e a percepção de suporte organizacional. Tese de doutorado não-publicada. Universidade de Brasília, Brasília.

Tamayo, M. R. (2003). Validação do Inventário de Burnout de Maslach. [Resumo]. In Universidade Federal da Paraíba (Org.), III Congresso Norte-Nordeste de Psicologia. Anais (p. 392-393). João Pessoa: UFPB.

Topf, M., \& Dillon, E. (1988). Noise-induced stress as a predictor of burnout in critical care nurses. Research in Nursing and Health, 12(3), 179-186.

Mauricio Robayo Tamayo, doutor em Psicologia pela Universidade de Brasília (UnB), é professor adjunto da Faculdade de Ceilândia (FCE) da mesma universidade.

Endereço para correspondência: SQN 412, Bloco N, Apto. 203, Brasília - DF. CEP 70867-140. Tel: (61) 3376-7487 / 3376-6042. E-mail: maurobayo@yahoo.com.br

Bartholomeu Torres Tróccoli, doutor em Psicologia Social e da Personalidade pela University of WisconsinMadison (EUA), é professor associado do Departamento de Psicologia Social, do Trabalho e das Organizações (PSTO) da Universidade de Brasília (UnB). E-mail: btroccoli@gmail.com 